\title{
Inequidades sociales y diabetes en adolescentes
}

\author{
Rodrigo López ${ }^{1}$, Vibeke Baelum². \\ Social inequities \\ and diabetes among adolescents
}

Background: Diseases are not randomly distributed across society. Although the results of several studies consistently report higher occurrence of diabetes among disadvantaged groups in adult populations, rather sparse and contradictory findings have been reported for the young. Aim: To explore whether the distribution of self reported diabetes among high school students from the Province of Santiago is associated to their socioeconomic position. Material and methods: Data originated from a randomly selected sample of 9,203 Chilean high school students. Students answered questionnaires on demographic variables; self reported diabetes status; and several dimensions of social position. We used logistic regression analyses to study the association between diabetic status and the variables 'number of cars owned by the family'; 'paternal income' (in Chilean pesos); and 'maternal' and 'paternal achieved education'. Results: Univariate analyses suggest the existence of social gradients for the variables number of cars owned and parental education. Multilevel analyses indicate that paternal achieved education is the most influential variable. Students coming from families in which the father has only primary school education, are significantly more likely to report having diabetes (odds ratio $=2.03$ confidence intervals 1.02-4.04). Conclusions: In this adolescent population, there is a positive association between several indicators of socioeconomic position and reported diabetes (Rev Méd Chile 2006; 134: 549-55).

(Key words: Diabetes mellitus; Social class; Socioeconomic factors)

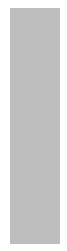

Recibido el 7 de marzo, 2005. Aceptado el 3 de octubre, 2005.
Estudio parcialmente financiado por el Danish Medical Research Con
mo no tuvo ninguna influencia en la planificación o ejecución
preparación, revisión o aprobación del manuscrito.
Faculty of Health Sciences, University of Aarhus, Aarhus, Denmark.
1DDS, PhD
2DDS, PhD, dr.odont

as enfermedades no se distribuyen aleatoreamente en la sociedad e individuos en posiciones de desventaja socioeconómica presentan un

Correspondencia a: Rodrigo López. Faculty of Health Sciences, University of Aarhus. Vennelyst Boulevard 9, DK8000 Aarhus C, Denmark. Tel: +45 89424141. Fax: +45 86136550. E mail: rlopez@odont.au.dk riesgo aumentado de sufrir numerosas patologías $^{1-6}$. Las explicaciones tentativas para esta asociación, sugieren que las inequidades sociales aumentan la exposición a factores dañinos y la susceptibilidad a sufrir enfermedades, así como también obstaculizan los recursos de protección, como el acceso a la educación en medidas de autocuidado, acceso a la atención médica oportuna y a un amplio espectro de oportunidades en la 
vida $^{1,2,7}$. Un claro ejemplo de esta situación en adultos es la diabetes. Numerosos estudios ${ }^{8-17}$ documentan la disminución del riesgo de presentar diabetes en adultos de condición socioeconómica favorable, sin embargo, los estudios disponibles sobre la posible relación entre la posición socioeconómica y la ocurrencia de diabetes en niños y adolescentes es escasa y sus resultados son controversiales ${ }^{18-22}$. Parte de esto puede deberse al uso de indicadores socioeconómicos inapropiados ${ }^{18,19}$. Paralelamente, los resultados de un número importante de estudios confirma que el control glicémico en niños y adolescentes diabéticos es considerablemente mejor en sujetos de mejor situación socioeconómi$\mathrm{ca}^{23-26}$, estos últimos hallazgos sugieren que, a nivel poblacional, es posible encontrar una mayor ocurrencia de diabetes precoz en sujetos en posición de desventaja socioeconómica. El objetivo de los análisis aquí reportados fue explorar si la distribución de diabetes reportada por estudiantes de enseñanza media, de una muestra poblacional de Santiago, se asocia a indicadores socioeconómicos de uso habitual en epidemiología.

\section{MATERIAL Y MÉTODO}

La información utilizada para estos análisis se originó de un estudio transversal de epidemiología oral multietápico, realizado en estudiantes de enseñanza media de la provincia de Santiago, Chile $^{27-29}$, diseñado para estudiar enfermedades periodontales en dicha población. El protocolo de investigación fue aprobado por el comité de ética local de la Universidad de Chile. La población blanco del estudio fue definida como 'todos los estudiantes asistiendo a enseñanza media en los colegios de la provincia de Santiago, Chile'. Para seleccionar la muestra poblacional del estudio obtuvimos la lista completa de establecimientos educacionales de enseñanza media de la provincia de Santiago (618 colegios) provista por el Ministerio de Educación de Chile. Dicho universo incluye aproximadamente $85 \%$ de la población adolescente de la provincia de Santiago.

Primera etapa de muestreo. Utilizando el programa SPSS $10^{30}$, separamos la lista de colegios en dos listas estratificadas, dependiendo de si los colegios recibían (333 colegios) o no recibían (285 colegios) soporte estatal. Cada una de ambas listas fue reordenada, utilizando el procedimiento 'random.exe' del programa estadístico PEPI para generar dos listas de colegios ordenadas en secuencia aleatoria ${ }^{31}$. El programa usa un algoritmo generador de números que ha sido extensivamente estudiado ${ }^{31}$. Las listas resultantes fueron permutadas alternadamente de modo de obtener una lista única, en que todos los colegios, con y sin soporte estatal, se alternaran uno a uno. Los directores de los primeros 133 colegios de la lista fueron contactados, para obtener información actualizada del número de estudiantes en cada uno de los 4 niveles de enseñanza media, el número de clases en cada colegio y su interés en participar en un proyecto epidemiológico de esta naturaleza. En 29 colegios, la información obtenida fue insuficiente para asegurar su inclusión en el estudio, resultando en 104 colegios potencialmente elegibles. De esos 104, los directores de 6 colegios declinaron de participar por tener un número importante de actividades extra programáticas en calendario, dejando un total de 98 colegios que fueron finalmente incluidos en el estudio 27 .

Segunda etapa de muestreo. El número de estudiantes por clases fue homogéneo, pero el número de estudiantes en cada nivel varió considerablemente entre los diferentes colegios y una segunda etapa de muestreo fue diseñada para incluir cantidades similares de estudiantes provenientes de cada colegio. En colegios donde el número total de estudiantes de enseñanza media era igual o menor a 100 individuos y en aquellos colegios donde el número de clases era de 3 o menor, todos los estudiantes presentes el día de la visita de los examinadores fueron invitados a participar en el estudio. En aquellos colegios de mayor tamaño, donde el número de estudiantes superaba los $100 \mathrm{y}$ el número de clases superaba las 3 clases, el procedimiento 'random.exe' del programa PEPI fue utilizado para generar una secuencia de clases ordenada aleatoriamente. Acto seguido, las 3 primeras clases de cada lista fueron seleccionadas para ser incluidas en el estudio; este procedimiento se repitió para cada colegio de mayor tamaño ${ }^{27}$.

Cada colegio fue visitado un día para realizar los exámenes. Un equipo de 2 examinadores 
visitó cada sala de clases e informó a los estudiantes sobre los objetivos del estudio, la naturaleza de los exámenes clínicos y sobre los cuestionarios que se realizarían en el colegio, los estudiantes fueron invitados a participar y se solicitó su aprobación individual antes de realizar cualquier tipo de examen o solicitud de responder cuestionarios. En la sala de clases, los alumnos recibieron una explicación general sobre diabetes. Todavía en la sala de clases, a aquellos estudiantes que aceptaron participar en el estudio, se les pidió que completaran un cuestionario con información sobre conductas relacionadas con salud e información demográfica general. El cuestionario incluyó una variable dicotómica sobre la condición de diabetes (eres diabético? SI/NO). Los estudiantes fueron instruidos para responder NO si ignoraban que fueran diabéticos. No se realizó ningún intento para determinar si el diagnóstico reportado había sido realizado por un médico o sobre el tipo de diabetes reportado. Un total de 9.203 estudiantes de 12 a 21 años de edad participó en el estudio. Cuarenta y un estudiantes quisieron responder el cuestionario, pero no quisieron participar en los exámenes clínicos o no pudieron ser examinados por otras razones.

Una vez finalizados los exámenes clínicos, los estudiantes recibieron el segundo cuestionario concerniente a distintas dimensiones de su posición socioeconómica. Los presentes análisis incluyen: número de automóviles de propiedad de la familia, ingreso mensual paterno en pesos chilenos y nivel de educación alcanzado por la madre y el padre.

Estadística. Utilizamos análisis de regresión logística para investigar la asociación entre los indicadores sociales seleccionados y el reporte de ser diabético, usando el programa estadístico STATA $9.0^{32}$. Realizamos análisis de regresión logística univariable para los 4 indicadores de posición socioeconómica estudiados y aquellas variables mostrando un valor de $p<0,25$ fueron seleccionadas para ser incluidas como covariables, en un modelo de regresión logística multivariable. La opción 'robust cluster' para el procedimiento 'logit' del programa Stata $9.0^{32}$ fue utilizada para ajustar por la unidad de muestreo 'clase' y el hecho de que estudiantes de una misma clase no pueden ser considerados independientes.
El modelo final fue construido a través de la exclusión consecutiva de una variable a la vez, utilizando el test de 'Likelihood ratio' descrito por Hosmer y Lemeshow ${ }^{33}$. Evaluamos la estabilidad del modelo después de cada modificación, verificando los cambios ocurridos en las estimaciones (odds ratio) después de eliminar cada variable ${ }^{33}$. Una vez obtenido el modelo final, evaluamos la posibilidad de interacción entre variables, introduciendo las combinaciones posibles de los indicadores estudiados, una a la vez como ha sido descrito por Hosmer y Lemeshow ${ }^{33}$.

\section{RESULTADOS}

Un total de 9.163 estudiantes de 12 a 21 años de edad, respondieron el cuestionario sobre dimensiones de posición socioeconómica. De ellos, 59 estudiantes señalaron ser diabéticos/as. Un estudiante no respondió a la pregunta de diabetes (Tabla 1).

La participación en las respuestas al cuestionario fluctuó entre 91,4\% para la pregunta sobre 'ingreso paterno' y 98,6\% para la pregunta sobre nivel de educación alcanzada por la madre ${ }^{34}$.

Para los indicadores 'número de automóviles de propiedad de la familia' y nivel de educación alcanzado por la madre y el padre los análisis de regresión logística univariables evidenciaron una tendencia a seguir gradientes sociales, indicando que sujetos cuyas familias no poseen automóvil $(\mathrm{OR}=1,89[0,90 ; 3,97])$ o poseen solamente un automóvil (OR=1,22 [0,54;2,71]); y estudiantes cuyo padre $(\mathrm{OR}=2,03 \quad[1,05 ; 3,93])$ o madre $(\mathrm{OR}=2,06[1,04 ; 4,10])$ han alcanzado menores niveles de educación, reportan con mayor frecuencia ser diabéticos (Tabla 2).

Los resultados del análisis de regresión multivariable revelaron que el nivel de educación alcanzado por el padre fue la variable más influyente en este estudio, con estudiantes cuyo padre solamente ha estudiado educación básica, reportando significativamente con más frecuencia la ocurrencia de diabetes $(\mathrm{OR}=2,03$ $[1,02 ; 4,04])$. Debido a colinearidad entre variables, fue imposible obtener un modelo multivariable que incluyera evaluación de interacción entre variables. 
Tabla 1. D istribución de los indicadores de posición socioeconómica y participación de los estudiantes. Se incluye el número de sujetos reportando diabetes en cada categoría

\begin{tabular}{|lcr|}
\hline & $\begin{array}{c}\text { Número de estudiantes } \\
\text { reportando diabetes }\end{array}$ & $\mathrm{n}$ \\
\hline Número de automóviles de la familia & & \\
No responde & 2 & 437 \\
Sin automóvil & 31 & 3.571 \\
1 automóvil & 18 & 3.208 \\
2 o más automóviles* & 8 & 1.947 \\
Total & 59 & 9.163 \\
Ingreso mensual del padre & & \\
No responde & 7 & 785 \\
Sin ingresos & 6 & 575 \\
<100.000 & 6 & 1.136 \\
\$100.000 - \$299.000 & 19 & 3.087 \\
\$300.000 - \$499.000 & 9 & 1.318 \\
>500.000* & 12 & 2.262 \\
Total & 59 & 9.163 \\
Nivel educacional alcanzado por el padre & & \\
No responde & 3 & 443 \\
Hasta educación primaria completa & 28 & 2.974 \\
Hasta enseñanza media completa & 16 & 2.955 \\
Hasta educación técnica o universitaria* & 12 & 2.791 \\
Total & 59 & 9.163 \\
Nivel educacional alcanzado por la madre & & \\
No responde & 1 & 124 \\
Hasta educación primaria completa & 32 & 3.464 \\
Hasta enseñanza media completa & 16 & 3.132 \\
Hasta educación técnica o universitaria* & 10 & 9.163 \\
Total & 59 & \\
\hline
\end{tabular}

*=1 estudiante no respondió el reporte de diabetes.

$\mathrm{n}=$ Número de estudiantes participantes.

\section{DisCUSIÓN}

Es una noción general que sujetos, en desventaja socioeconómica absoluta, presentan con mayor frecuencia un número importante de patologías en relación al resto de los miembros de una sociedad $^{1-3}$. Sin embargo, el problema de inequidades sociales en salud es más complejo y el efecto de la posición de los sujetos en la ocurrencia y severidad de muchas enfermedades, parece no restringirse a individuos en grupos caracterizados por deprivación severa o absoluta, sino que se manifiesta a distintos niveles de las jerarquías sociales, generando lo que hoy se conoce como gradiente social en salud 2,6,35,36. Los resultados del presente estudio revelan que la posición socioeconómica relativa de los adolescentes participantes, se asocia a la forma en que reportan la ocurrencia de diabetes; es decir, la frecuencia de diabetes autorreportada para cada categoría parece ser mayor que la reportada por sujetos en la categoría inmediatamente superior.

Es importante reconocer la existencia de estos gradientes sociales en la ocurrencia de algunas 
Tabla 2. Análisis de regresión logística de la asociación entre indicadores socioeconómicos
y reporte de diabetes con los respectivos intervalos de confianza (95\%)

\begin{tabular}{|c|c|c|c|c|c|}
\hline \multirow[t]{2}{*}{ Indicadores } & \multicolumn{3}{|c|}{ Univariable } & \multicolumn{2}{|c|}{ Modelo final } \\
\hline & OR & $95 \%$ CI & $\mathrm{p}$ & OR & $95 \% \mathrm{CI}$ \\
\hline \multicolumn{6}{|l|}{ № de automóviles de la familia } \\
\hline 2 o más automóviles (ref) & 1 & - & & - & - \\
\hline 1 automóvil & 1,22 & {$[0,54 ; 2,71]$} & 0,63 & - & - \\
\hline Sin automóvil & 1,89 & {$[0,90 ; 3,97]$} & 0,09 & - & - \\
\hline No responde & 0,99 & {$[0,21 ; 4,60]$} & 0,99 & - & - \\
\hline \multicolumn{6}{|l|}{ Ingreso mensual del padre } \\
\hline$\geq \$ 500.000$ (ref) & 1 & - & & - & - \\
\hline$\$ 300-\$ 499.000$ & 1,19 & {$[0,51 ; 2,79]$} & 0,69 & - & - \\
\hline$\$ 100-\$ 299.000$ & 1,07 & {$[0,53 ; 2,17]$} & 0,85 & - & - \\
\hline$<\$ 100.000$ & 0,92 & {$[0,35 ; 2,42]$} & 0,86 & - & - \\
\hline Sin ingresos & 1,82 & {$[0,69 ; 4,82]$} & 0,23 & - & - \\
\hline No responde & 1,56 & {$[0,62 ; 3,92]$} & 0,35 & - & - \\
\hline \multicolumn{6}{|l|}{ Nivel educacional alcanzado por el padre } \\
\hline Educación técnica o universitaria (ref) & 1 & - & & - & - \\
\hline Enseñanza media completa & 1,16 & {$[0,56 ; 2,42]$} & 0,69 & 1,16 & {$[0,54 ; 2,51]$} \\
\hline Hasta educación primaria completa & $2,03^{\#}$ & {$[1,05 ; 3,93]$} & 0,04 & $2,03^{\#}$ & {$[1,02 ; 4,04]$} \\
\hline No responde & 1,46 & {$[0,41 ; 5,13]$} & 0,56 & 1,46 & {$[0,44 ; 4,82]$} \\
\hline \multicolumn{6}{|l|}{ Nivel educacional alcanzado por la madre } \\
\hline Educación técnica o universitaria (ref) & 1 & - & & - & - \\
\hline Enseñanza media completa & 1,14 & {$[0,53 ; 2,45]$} & 0,75 & - & - \\
\hline Hasta educación primaria & $2,06^{\#}$ & {$[1,04 ; 4,10]$} & 0,04 & - & - \\
\hline No responde & 1,80 & {$[0,23 ; 14,03]$} & 0,58 & - & - \\
\hline
\end{tabular}

\#= Estadísticamente significativo. OR= Odds ratio. 95\% CI=95\% intervalo de confianza

Debido a colinearidad entre las variables, fue imposible incluir control por interacción en el modelo final.

enfermedades, pues este enfoque puede proveer información sobre la etiología de las enfermedades que no es factible obtener cuando la investigación está dirigida, exclusivamente, a elucidar las causas aparentemente obvias de enfermedad en la asociación dicotómica extrema pobreza/no pobreza ${ }^{36,37}$.

El nivel educacional alcanzado por los padres puede disminuir el riesgo de exposición a factores dañinos y reforzar mecanismos protectivos. El ingreso paterno y la medida del número de vehículos, usado como un indicador de prosperidad, pueden representar acceso preferencial a atención médica oportuna en la sociedad chilena. Debido a la esperable relación entre diversas dimensiones de posición social no fue posible desarrollar un modelo multivariable, que incluyera la ocurrencia de interacción entre indicadores. Esto es esperado, considerando que a pesar de que diversos aspectos de la posición social de los individuos contribuyen con distintos aspectos de la vida, la relación entre los indicadores hace difícil una interpretación coherente de dichos modelos.

Una de las posibles limitaciones de este estudio es la falta de información sobre la validez del diagnóstico de diabetes empleado. Resultados de previos estudios en adultos ${ }^{38-40}$ indican que el reporte de diabetes puede ser un indicador apropiado, sin embargo, no existe información sobre la validez de dicho indicador en la población estudiada. Si los hallazgos presentados reflejan la distribución de diabetes en esta población, resulta particularmente preocupante que estos gradientes sociales se evidencien a una edad tan temprana. 


\section{REFERENCIAS}

1. Lynch J, Kapian G. Socioeconomic position. En: Berkman LF, Kawachi I, ed. Social epidemiology. New York: Oxford University press, Inc, 2000; 13-35.

2. Marmot M, Feeney A. General explanations for social inequalities in health. IARC Sci Publ 1997; 138: 207-28.

3. Bartley M, Blane D, Brunner E, Mccarthy M, Dorling D, FerRIE J ET al. Social determinants of health. The solid facts. Copenhagen: Centre for Urban Health World Health Organization Regional Office for Europe; 1998.

4. Adier N, Marmot M, McEwen B, Stewart J. Eds. Socioeconomic status and health in industrial nations: social, psychological, and biological pathways. N York: New York Academy of Sciences; 1999.

5. BerkMan LF, KaWachi I. Social epidemiology. $1^{\text {st }}$ ed. New York: Oxford University Press; 2000.

6. GoLdman N. Social inequalities in health. Disentangling the underlying mechanisms. Ann NY Acad Sci 2001; 954: 118-39.

7. BerkMan LF, Macintyre $S$. The measurement of social class in health studies: old measures and new formulations. IARC Sci Publ 1997; (138): 51-64.

8. Self-reported prevalence of diabetes among Hispanics- United States, 1994-1997. MMWR 1999; 48: 8-12.

9. Goyder E, Hammersiey V. Explaining variations in reported diabetes prevalence in general practice: how much variation is explained by differences between practice populations? Br J Gen Pract 2003; 53: 642-4.

10. BeEChing NJ, GiL GV. Deprivation and type 2 diabetes mellitus prevalence. Diabet Med 2000; 17: 813-5.

11. Evans JMM, Newton RW, Ruta DA, MacDonald TM, Morris AD. Socio-economic status, obesity and prevalence of type 1 and type 2 diabetes. Diabet Med 2000; 17: 478-80.

12. Connowy V, Unwin N, Sherrify P, BiLous R, Ke山y W. Diabetes prevalence and socioeconomic status: a population based study showing increased prevalence of type 2 diabetes mellitus in deprived areas. J Epidemiol Community Health 2000; 54: 173-7.

13. Brancati FL, Whelton PK, Kuluer LH, Kiag MJ. Diabetes mellitus, race, and socioeconomic status. A population-based study. Ann Epidemiol 1996; 6: 67-73.
14. Agardh EE, Ahlbom A, Andersson A, EFendic S, GRIL V, HALQVIST J ET al. Explanations of socioeconomic differences in excess risk of type 2 diabetes in Swedish men and women. Diabetes Care 2004; 27: 716-21.

15. Kaufman JS, Durazo-Arvizu R, McGee DL, Cooper RS. Letter. Ann Epidemiol 1997; 7: 76-7.

16. Ruste L, Khan F, CRUickshank K. High prevalence of type 2 diabetes in all ethnic groups, including Europeans, in a British inner city. Diabetes Care 2001; 24: 1377-83.

17. Glover JD, Hetzel DMS, Tennant SK. The socioeconomic gradient and chronic illness and associated risk factors in Australia. Aust New Zealand Health Policy 2004; 1: 8.

18. La Porte RE, Orchard TJ, KuLer LH, Wagener DK, DRASH AL, SCHNEIDER BB ET AL. The Pittsburgh insulin dependent diabetes mellitus registry. The relationship of insulin dependent diabetes mellitus incidence to social class. Am J Epidemiol 1981; 114: 379-84.

19. Siemiatycki J, Cole E, Campbell S, Dewar R, Aubert D, BELMONTE MM. Incidence of IDDM in Montreal by ethnic group and social class and comparisons with ethnic groups living elsewhere. Diabetes 1988; 37: 1096-102.

20. Patterson CC, Waugh NR. Childhood diabetes and material deprivation. Br Med J 1991; 303: 523.

21. Crow YJ, Alberti KGMM, ParKIN JM. Insulin dependent diabetes in childhood and material deprivation in northern England, 1977-86. Br Med J 1991; 303: 158-60.

22. Singh R, Shaw J, Zimmet P. Epidemiology of childhood type 2 diabetes in the developing world. Pediatr Diabetes 2004; 5: 154-68.

23. Delamater AM, Albrecht DR, Poste山on DC, Gutai JP. Racial differences in metabolic control of children and adolescents with type I diabetes mellitus. Diabetes Care 1991; 14: 20-5.

24. OverstreEt S, Holmes CS, Dunlap WP, Frentz J. Sociodemographic risk factors to disease control in children with diabetes. Diabet Med 1997; 14: 153-7.

25. Delamater AM, Shaw KH, Applegate EB, Pratt iA, Eidson M, Lancelotta GX et aL. Risk for metabolic control problems in minority youth with diabetes. Diabetes Care 1999; 22: 700-5.

26. Gauiegos-Macias AR, Macias SR, Kaufman E, Skipper B, KaLSHMAN N. Relationship between glycemic control, ethnicity and socioeconomic status in Hispanic and white Non-Hispanic youths with type I diabetes mellitus. Pediatr Diabetes 2003; 4: 19-23. 
27. López R, Fernández O, Jara G, Baelum V. Epidemiology of clinical attachment loss in adolescents. J Periodontol 2001; 72: 1666-74.

28. LóPeZ R. Periodontitis in adolescents. Studies among Chilean high school students. Denmark Royal Dental College, Faculty of Health Sciences, University of Aarhus; Faellestrykkeriet for Sundhedsvidenskab. Aarhus 2003.

29. Lopez R, Fernández O, Jara G, Baelum V. Epidemiology of necrotizing ulcerative gingival lesions in adolescents. J Periodont Res 2002; 37: 439-44.

30. SPSS [computer program]. Version 10.0. SPSS Inc. Headquarters, $233 \mathrm{~S}$. Wacker Drive, 11th floor, Chicago, Illinois 60606: 2000.

31. Computer programs for epidemiologists. PEPI 3.01 [computer program]. Llanidloes, UK: Brixton Books; 2000.

32. StataCorp. Statistical Software: Release 9.0 [computer program]. College Station, TX: Stata Corporation; 2005.

33. Hosmer DW, Lemeshow S. Applied logistic regression. $2^{\text {nd }}$ ed. New York: John Wiley \& Sons; 2000.

34. Lopez R, Fernández O, Baelum V. Social gradients in periodontal disease among adolescents. Community Dent Oral Epidemiol. 2006; 34: 184-96.

35. Adler NE, Ostrove JM. Socioeconomic status and health: what we know and what we don't. Ann NY Acad Sci 1999; 896: 3-15.
36. Starfield B, Riley AW, Witt WP, Robertson J. Social class gradients in health during adolescence. J Epidemiol Community Health 2002; 56: 354-61.

37. Manor O, Matthews S, Power C. Companing measures of health inequality. Soc Sci Med 1997; 45: 761-71.

38. Trends in the prevalence and incidence of selfreported diabetes mellitus - United States, 19801994. MMWR 1997; 46: 1014-8.

39. Midthjell K, Holmen J, BJørndal A, Lund-Larsen PG. Is questionnaire information valid in the study of a chronic disease such as diabetes? The NordTrøndelag diabetes study. J Epidemiol Community Health 1992; 46: 537-42.

40. OkURa Y, Urban LH, Mahoney DW, Jacobsen SJ, RODEHEFFER RJ. Agreement between self-report questionnaires and medical record data was substantial for diabetes, hypertension, myocardial infarction and stroke but not for heart failure. J Clin Epidemiol 2004; 57: 1096-103.

\section{Agradecimientos}

La recomendación de utilizar varios indicadores sociales y la revisión de los posibles candidatos para ser usados en el estudio por parte de la Asistente Social Sra. Marta Valdenegro es muy apreciada y agradecida por los autores. 